\title{
MPPT Algorithm for Photovoltaic Panel Based on Augmented Takagi-Sugeno Fuzzy Model
}

\author{
Hafedh Abid, Ahmed Toumi, and Mohamed Chaabane \\ Laboratory of Sciences and Techniques of Automatic Control \& Computer Engineering (Lab-STA), \\ National School of Engineering of Sfax, University of Sfax, P.O. Box 1173, 3038 Sfax, Tunisia
}

Correspondence should be addressed to Ahmed Toumi; toumi.a@gmail.com

Received 30 October 2013; Accepted 19 December 2013; Published 17 February 2014

Academic Editors: T.-H. Meen and R. Miles

Copyright (c) 2014 Hafedh Abid et al. This is an open access article distributed under the Creative Commons Attribution License, which permits unrestricted use, distribution, and reproduction in any medium, provided the original work is properly cited.

This paper deals with the Maximum Power Point Tracking (MPPT) for photovoltaic energy system. It includes photovoltaic array panel, DC/DC converter, and load. The operating point for photovoltaic energy system depends on climatic parameters and load. For each temperature and irradiation pair, there exists only one optimal operating point which corresponds to the maximum power transmitted to the load. The photovoltaic energy system is described by nonlinear equations. It is transformed into an augmented system which is described with a Takagi-Sugeno (T-S) fuzzy model. The proposed MPPT algorithm which permits transfering the maximum power from the panel to the load is based on Parallel Distributed Compensation method (PDC). The control parameters have been computed based on Linear Matrix Inequalities tools (LMI). The Lyapunov approach has been used to prove the stability of the system. Some reliable simulation results are provided to check the efficiency of the proposed algorithm.

\section{Introduction}

In the most recent years, photovoltaic (PV) energy has been the subject of several research projects. It is well known that the PV array power panel depends on climatic variables such as temperature and irradiation as shown in Figures 2 and 3. Actually, the operating point of the PV array panel depends on three parameters such as temperature, irradiation, and the load. In fact, the operating point results from the intersection of the I-V characteristic and the load characteristic as shown in Figure 4. In most cases, the value of load is constant and the climatic parameters vary in the day, so the load characteristic remains fixed and the characteristic of the panel varies according to climatic variables. Consequently, the operating point is variable and the load cannot extract maximum power from the panel. To overcome this disadvantage, a DC/DC converter is inserted between the panel and the load. In this way, the load value seen by the PV panel can be changed by varying the duty cycle. In this context, several studies have been developed.

Most of papers dealing with the MPPT control algorithms are based on perturb and observe ( $\mathrm{P} \& \mathrm{O})$ [1-4], Incremental Conductance $[3,5]$, Mamdani type fuzzy logic controller
(FLC) $[4,6]$, and some different approaches as neural network controller (NNC) [7]. In this paper, the PV array panel has been modelled by fuzzy system approach. At every time, the desired state variables have been computed based on the measurement of temperature and irradiation. Also, the MPP tracker algorithm has been developed based on Parallel Distributed Compensation (PDC) method which was designed for fuzzy system. Most of papers which have used fuzzy system applied Mamdani method. Whereas in this work, the main contribution which deals with Maximum Power Point Tracking for photovoltaic panel, consists on developing a new algorithm based on augmented T-S fuzzy system. It is well known that the Mamdani fuzzy system includes three blocks which are fuzzification, fuzzy inference rules and defuzzification, whereas the T-S fuzzy system needs only two blocks such as fuzzification and fuzzy inference rules. The block number has been reduced. However, we decided to choose the T-S type fuzzy system. In this paper, the T-S fuzzy system has been used in modelling stage of PV system and in the control stage. The optimal duty cycle, which permits extracting the maximum power from the photovoltaic array panel, is computed based on PDC techniques. This paper is organised as follows. In Section 2, 


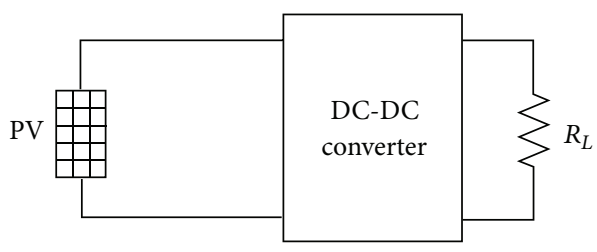

FIgure 1: Photovoltaic system.

we describe the problem statement and we show the influence of climatic parameters such as temperature and irradiation on the electrical characteristic of the PV array panel. Then, we recall the model of photovoltaic panel. At the end of the second section, we describe the photovoltaic energy system by a state model. The third section is reserved to present the control strategy. In the first part, we recall the T-S fuzzy system; then we describe the photovoltaic energy system by an augmented T-S fuzzy model. In the second part of the third section, we describe the T-S fuzzy reference model and the MPPT algorithm which is based on T-S fuzzy system.

Section four is devoted to the stability analysis of the closed loop system. The feedback gains have been computed by solving LMIs expressions. The simulation results of photovoltaic energy system showing performances of the proposed MPPT algorithm tracker are discussed in Section 5. Conclusions are drawn in the final section.

\section{Problem Statement}

The photovoltaic power depends on climatic parameters such as temperature and irradiation as shown in Figures 2 and 3. In fact, the photovoltaic power, which is transmitted to the load, is function of the impedance of the load and the climatic parameters as shown in Figure 4. However, to change the impedance seen by the panel, it is necessary to insert a DC/DC converter. The photovoltaic system consists of a photovoltaic array panel connected to a DC-DC converter which provides energy to the load, as shown in Figure 1.

Figures 2 and 3 show the evolution of the generated power curves as a function of voltage, respectively, for a given constant irradiation and different values of temperature and then for a given constant temperature and different values of irradiation.

In conclusion, we can say that the PV array panel is nonlinear and time-variant system. From Figures 2 and 3, it is clear that the temperature affects essentially the voltage and the irradiation affects fundamentally the intensity of the PV array panel. Also, we can conclude that the output power generated by the PV array panel depends on the climatic parameters " $G$ and $T$." In fact, the power increases with an increase in solar radiation and decreases with an increase in temperature. For each given pair of parameters $(G, T)$, there exists only one Maximum Power Point (MPP). The operating point is determined by the intersection of the panel current-voltage characteristic and the load current-voltage characteristic.

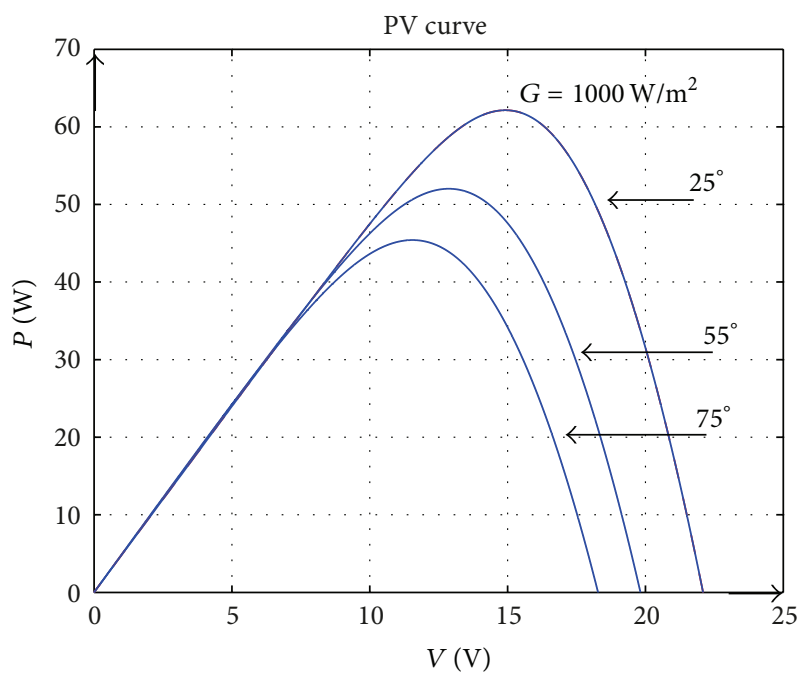

FIgURE 2: PV power curves with different $T$.

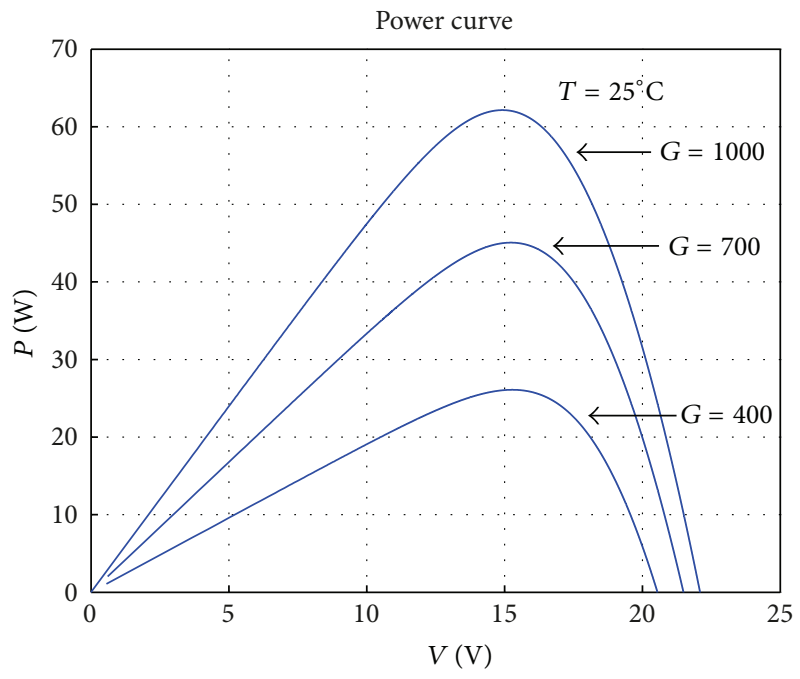

Figure 3: PV power curves with different $G$.

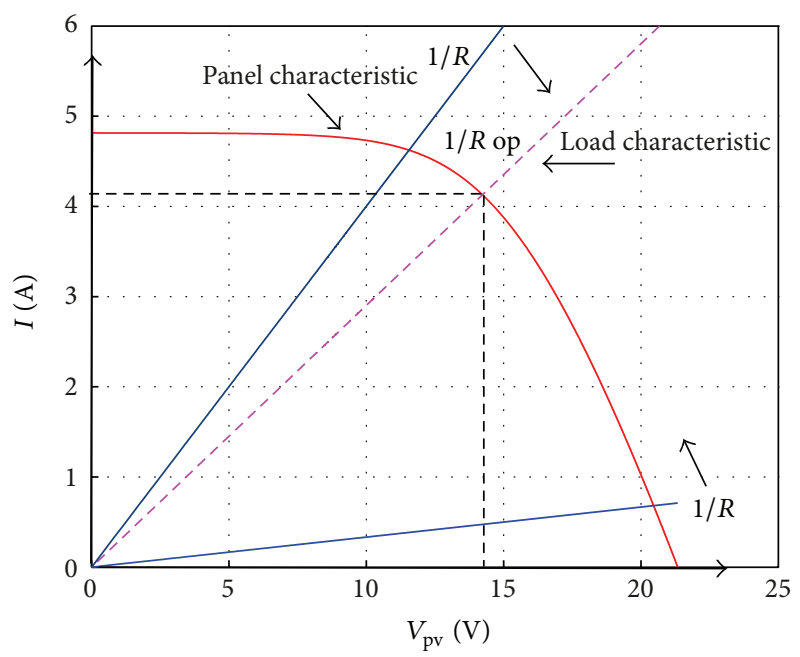

FIGURE 4: Operating point of the PV array panel. 


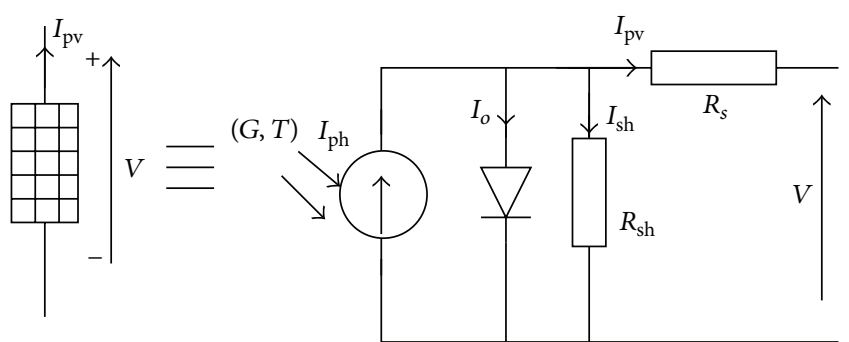

FIgURE 5: Equivalent circuit for PV cell.

However, a specific algorithm tracker should be used to search the optimal operating point which permits to extract the maximum power from the PV array panel.

In the next part of this section, we recall the most popular model which is developed by Singer et al. [8]; then we describe the modeling of the overall photovoltaic system energy.

The electrical equivalent circuit of the PV cell is given by Figure 5.

It consists of a current generator which depends on irradiation $(G)$ and temperature $(T)$, in parallel with a diode, and connected to an internal parallel and series resistor, namely, respectively, $R_{\mathrm{sh}}$ and $R_{s}$.

The PV cell model is described by the following equations:

$$
\begin{gathered}
I_{\mathrm{sh}}=\frac{V+R_{s} I_{\mathrm{pv}}}{R_{\mathrm{sh}}}, \\
I_{\mathrm{pv}}=I_{\mathrm{ph}}-I_{o}\left[\exp \left(\frac{V+R_{s} I}{V_{t}}\right)-1\right]-\frac{\left(V+R_{s} I\right)}{R_{\mathrm{sh}}}, \\
V_{t}=\frac{n_{s} k T}{q} .
\end{gathered}
$$

The generated current by the photovoltaic panel varies with temperature and irradiation; its expression is given by the following equation:

$$
I_{\mathrm{ph}}=\left(I_{\mathrm{ph}, n}+K_{I} \Delta T\right) \frac{G}{G_{n}} .
$$

$I_{\mathrm{ph}, n}$ is the rated current generated by the PV panel under standard condition of temperature and irradiation $\left(T=25^{\circ} \mathrm{C}\right.$ and $G=1000 \mathrm{w} / \mathrm{m}^{2}$ ):

$$
I_{o}=\frac{\left(I_{\mathrm{ph}}+K_{I} \Delta T\right)}{\exp \left(\left(V_{\mathrm{oc}}+K_{v} \Delta T\right) / V_{t}\right)-1},
$$

where $I_{o}$ is a reverse saturation current:

$$
\begin{gathered}
V_{\mathrm{oc}}=n_{s} \frac{K T}{q} \log \left(\frac{I_{\mathrm{sc}}+I_{o}}{I_{o}}\right), \\
V_{c}=n_{s} \frac{K T}{q} \log \left(\frac{I_{\mathrm{sc}}+I_{o}-I_{\mathrm{pv}}}{I_{o}}\right),
\end{gathered}
$$

where $V_{\mathrm{oc}}$ is the open circuit voltage and $I_{\mathrm{sc}}$ is the short circuit current.
The overall photovoltaic system energy can be represented by the scheme illustrated in Figure 6.

The average dynamic model of the photovoltaic system given by Figure 6 can be expressed in continuous conduction by the following equations:

$$
\begin{gathered}
\frac{d V_{\mathrm{pv}}}{d t}=\frac{1}{C_{1}}\left(I_{\mathrm{pv}}-I_{L}\right), \\
\frac{d I_{L}}{d t}=\frac{1}{L}\left[V_{\mathrm{pv}}-V_{c 2}(1-\mu)\right], \\
\frac{d V_{c 2}}{d t}=\frac{1}{C_{2}}\left[I_{L}(1-\mu)-\frac{V_{c 2}}{R_{L}}\right] .
\end{gathered}
$$

In the continuous conduction, the average value of $I_{\mathrm{PV}}$ current is equal to the average value of $I_{L}$ current. of

It is very clear that the system can be described as the form

$$
\dot{x}(t)=A(x, t) x(t)+B u(t),
$$

where $x(t)=\left[\begin{array}{lll}V_{\mathrm{pv}} & I_{L} & V_{c 2}\end{array}\right]^{T}$ is the state vector, $A(x, t)$ is the state matrix, $B$ is the input vector, and $\mu$ is the duty ratio. However, the state matrix is nonlinear.

\section{Control Strategy}

The control strategy that we propose is given by Figure 7.

The control strategy consists of three blocks: reference model, controller, and plant, which is defined by PV system (see Figure 1).

3.1. T-S Fuzzy Model. Several studies have been proving that the Takagi-Sugeno fuzzy system can describe the behavior of continuous nonlinear system. However, we use in this work the T-S fuzzy system to describe the nonlinear energy conversion system. The fuzzy model is described by fuzzy rules where each rule represents input-output relations of linear local model. The $i$ th rule of the fuzzy model has the following form:

$$
\begin{aligned}
& \text { IF } z_{1} \text { is } M_{i 1} \text { and } z_{2} \text { is } M_{i 2} \text { and, } \ldots, \text { and } z_{n} \text { is } M_{i n} \\
& \text { THEN }\left\{\begin{array}{l}
\dot{x}(t)=A_{i} x(t)+B_{i} u(t), \\
y(t)=C_{i} x(t), \quad i=1,2, \ldots, c,
\end{array}\right.
\end{aligned}
$$

where $\left\{M_{i j}\right\}$ are the fuzzy sets, $x(t)$ is the state vector, $u(t)$ is the input vector, $A_{i}$ is the state matrix, $B_{i}$ is the input matrix, $z_{1}(t), \ldots, z_{n}(t)$ are the premise variables, $x(t) \in R^{n}$, $A_{i} \in R^{n \times n}, B_{i} \in R^{n x m}, y(t) \in R^{m}$, and $c$ is the number of fuzzy rules. The global fuzzy model of the system has the following form:

$$
\dot{x}(t)=\frac{\sum_{i=1}^{c} w_{i}(z(t))\left[A_{i} x(t)+B_{i} u(t)\right]}{\sum_{i=1}^{c} w_{i}(z(t))} .
$$




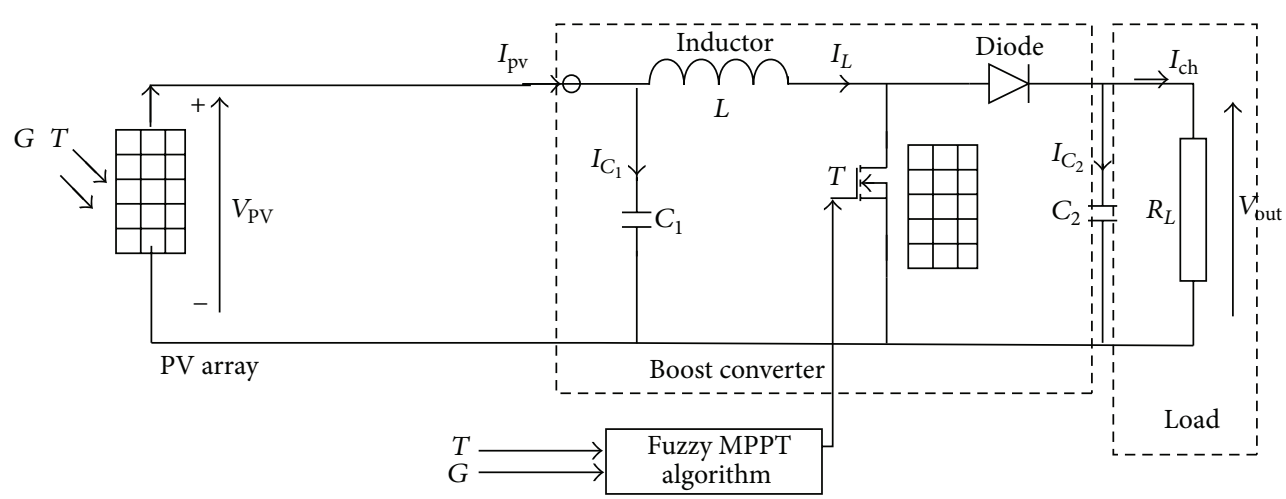

FIgure 6: Photovoltaic system.

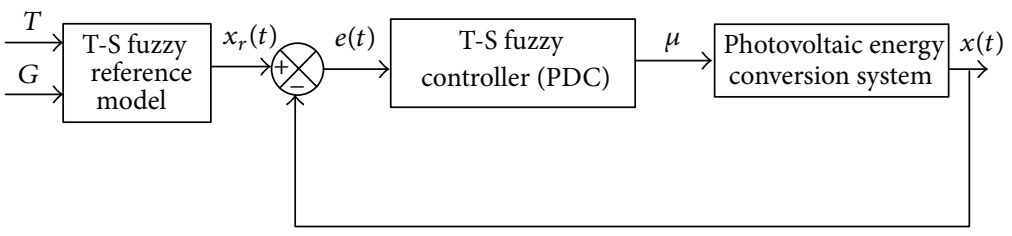

FIGURE 7: Control strategy.

For each rule $R_{i}$ attributed a weight $w_{i}(z(t))$ which depends on grade of membership function of premise variables $z_{j}(t)$ in fuzzy sets $M_{i j}$ :

$$
\begin{aligned}
& w_{i}(z(t))=\prod_{j=1}^{n} M_{i j}\left(z_{j}(t)\right), \quad w_{i}(z(t)) \geq 0, \\
& \sum_{i=1}^{c} w_{i}(z(t))>0, \quad \text { for } i=1, \ldots, c .
\end{aligned}
$$

$M_{i j}\left(z_{j}(t)\right)$ is the grade of membership of $z_{j}(t)$ to the fuzzy set $M_{i j}$ :

$$
\begin{gathered}
h_{j}(z(t))=\frac{w_{j}(z(t))}{\sum_{i=1}^{c} w_{i}(z(t))}, \quad 0 \leq h_{i}(z(t)) \leq 1, \\
\sum_{i=1}^{c} h_{i}(z(t))=1, \quad i=1, \ldots, c .
\end{gathered}
$$

The polytopic form of state equation is

$$
\dot{x}(t)=\sum_{i=1}^{c} h_{i}(z(t))\left[A_{i} x(t)+B_{i} u(t)\right] .
$$

3.2. Augmented T-S Fuzzy Model of Photovoltaic System. In the first stage of this subsection, we transform the average dynamic model of the photovoltaic system described by (5) into an augmented model. However, a new state variable must be added to the state vector. In other words an integrator is included previous to the real input $\mu$ and let $\dot{\mu}=u$. Therefore, $\mu$ becomes new state variable and $u$ is the new control input of the augmented system. Then, the system can be described as a nonlinear system in the form of

$$
\dot{x}(t)=A(x, t) x(t)+B u(t),
$$

where $x(t)=\left[\begin{array}{llll}V_{\mathrm{pv}} & I_{L} & V_{c 2} & \mu\end{array}\right]^{T}$ is the state vector, $A(x, t)$ is the state matrix, and $B$ is the input matrix:

$$
A=\left[\begin{array}{cccc}
\frac{\alpha}{C_{1}} & -\frac{1}{C_{1}} & 0 & 0 \\
\frac{1}{L} & 0 & -\frac{1}{L} & \frac{\beta}{L} \\
0 & \frac{1}{C_{2}} & \frac{-1}{R_{L} C_{2}} & -\frac{\gamma}{C_{2}} \\
0 & 0 & 0 & 0
\end{array}\right], \quad B=\left[\begin{array}{l}
0 \\
0 \\
0 \\
1
\end{array}\right] .
$$

with $\alpha=I_{\mathrm{pv}} / V_{\mathrm{pv}}, \beta=V_{c 2}$, and $\gamma=I_{L}$.

It is clear that (12) is nonlinear. To obtain the T-S fuzzy model, we choose the following three premises variables: $I_{\mathrm{PV}}$, $V_{\mathrm{PV}}$, and $V_{c 2}$. However, eight local models have been obtained to describe the T-S fuzzy model. The state matrix of each local model has the following structure:

$$
A_{i}=\left[\begin{array}{cccc}
\frac{\alpha_{i}}{C_{1}} & -\frac{1}{C_{1}} & 0 & 0 \\
\frac{1}{L} & 0 & -\frac{1}{L} & \frac{\beta_{i}}{L} \\
0 & \frac{1}{C_{2}} & \frac{-1}{R_{L} C_{2}} & -\frac{\gamma_{i}}{C_{2}} \\
0 & 0 & 0 & 0
\end{array}\right]
$$

where each of the variables $\alpha_{i}=I_{\mathrm{pvi}} / V_{\mathrm{pvi}}, \beta_{i}=V_{c 2 i}$, and $\gamma_{i}=I_{L i}$ must be replaced, respectively, by the appropriate value according to fuzzy rule base $\left(\alpha_{\min }\right.$ or $\left.\alpha_{\max }\right),\left(\beta_{\min }\right.$ or $\left.\beta_{\max }\right)$, and $\left(\gamma_{\min }\right.$ or $\left.\gamma_{\max }\right)$.

3.3. Reference Model. It is well known that the maximum power produced by panel, also the corresponding optimal 


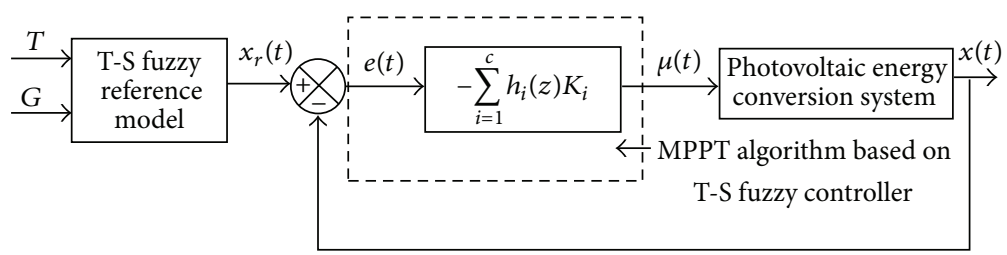

FIgURE 8: Control strategy of MPPT.

tension $V_{\mathrm{MP}}$ and optimal current $I_{\mathrm{MP}}$, depends on temperature $T$ and irradiation $G$. However, these latest are used as premise variables, $Z_{R 1}=T$ and $Z_{R 2}=G$, to compute the reference model based on T-S fuzzy system. The $i$ th rule of the fuzzy reference model has the following form:

$$
\begin{aligned}
& \text { IF } z_{1 R} \text { is } \lambda_{i 1} \text { and } z_{2 R} \text { is } \lambda_{i 2} \text { and, } \ldots, \text { and } z_{n R} \text { is } \lambda_{i n} \\
& \text { THEN }\left\{\begin{array}{l}
\dot{x}_{R}(t)=D_{i} x_{R}(t), \\
y_{R}(t)=C_{R i} x_{R}(t), \quad i=1,2, \ldots, c r,
\end{array}\right.
\end{aligned}
$$

where $\left\{\lambda_{i j}\right\}$ are the fuzzy sets, $x_{R}(t)=\left[\begin{array}{llll}V_{\mathrm{pvr}} & I_{L r} & V_{c 2 r} & \mu_{r}\end{array}\right]^{T}$ is the state reference variable vector, $D_{i} \in R^{n \times n}$ is the local reference state matrix, and $\left\{z_{1 R}(t), \ldots, z_{n R}(t)\right\}$ are the premise variables. $y(t) \in R^{m}$ is the output vector, $\mathrm{cr}$ is the number of fuzzy rules. Then, the T-S fuzzy reference model is given by the following equation:

$$
\dot{x}_{R}(t)=\sum_{i=1}^{c r} \eta_{i}(z(t)) D_{i} x_{R}(t)
$$

with $\sum_{i=1}^{c r} \eta_{i}(z(t))=1$ and $D_{i}$ is the local reference state matrix:

$$
D_{i}=\left[\begin{array}{cccc}
\frac{\delta_{i}}{C_{1}} & -\frac{1}{C_{1}} & 0 & 0 \\
\frac{1}{L} & 0 & -\frac{1}{L} & \frac{\sigma_{i}}{L} \\
0 & \frac{1}{C_{2}} & \frac{-1}{R_{L} C_{2}} & -\frac{\zeta_{i}}{C_{2}} \\
\frac{1}{1-\mu_{\mathrm{op} i}} & 0 & -1 & 0
\end{array}\right]
$$

with $\zeta_{i}=I_{\mathrm{MPP} i}, \delta_{i}=\zeta_{i} / V_{\mathrm{MPP} i}, \mu_{\mathrm{op} i}=1-1 / \sqrt{R_{L} \delta_{i}}$, and $\sigma_{i}=$ $V_{\mathrm{MPP} 2 i}=V_{\mathrm{MPP} i} /\left(1-\mu_{\mathrm{op} i}\right)$.

3.4. MPPT Algorithm Based on T-S Fuzzy Model. The crucial function of the MPPT algorithm is to search the coordinates of the optimal operating point. In this work, the MPPT algorithm provided at each time the appropriate duty ratio $\mu$ based on the desired and measured state variables. However, the MPPT algorithm which represents a controller is based on Parallel Distributed Compensation (PDC) method. The T-S fuzzy controller is designed from the T-S fuzzy model of photovoltaic energy conversion system. The T-S fuzzy controller is based on Parallel Distributed Compensation (PDC) technique, which is proposed by Wang et al. [9]. The particularity of the PDC controller is that it shares the same fuzzy sets in the premise parts with the fuzzy model. Based on the T-S fuzzy models, the PDC fuzzy controller is designed as follows.

ith controller rule:

$$
\begin{aligned}
& \text { IF } z_{1} \text { is } M_{i 1} \text { and } z_{2} \text { is } M_{i 2} \text { and, } \ldots, \text { and } z_{n} \text { is } M_{i n} \\
& \text { THEN } u(t)=-K_{i} x(t), \quad i=1,2, \ldots, c .
\end{aligned}
$$

The global fuzzy controller is represented by

$$
u(t)=-\sum_{i=1}^{c} h_{i}(z) K_{i} x(t)
$$

\section{Stability Analysis}

In the previous section, we discussed the main blocks of the control strategy and we have developed an MPPT algorithm (Figure 8). However, the system of energy conversion transmits the maximum PV generated power to the load and it is crucial to ensure that the closed-loop system is stable.

Theorem 1. Consider the reference model (16) which is used to compute the reference state variables, the nonlinear system (5) which can be modeled by the T-S fuzzy model (11), and the MPPT algorithm (19) based on the PDC techniques. If there exist a common symmetric positive definite matrix $Q$ and a feedback gains $K_{i}$ which satisfy the following LMIs (20), then the closed loop system is asymptotically stable and the tracking error converges toward zero:

$$
\begin{gathered}
{\left[\begin{array}{cc}
Q A_{i}^{T}+A_{i} Q-B_{i} M_{i}-M_{i}^{T} B_{i}^{T} & \left(A_{i}-D_{k}\right) Q \\
Q\left(A_{i}-D_{k}\right)^{T} & -\rho^{2} I
\end{array}\right]<0,} \\
\text { for } i=1, \ldots, c, k=1, \ldots, c r, \\
{\left[\begin{array}{cc}
Q A_{i}^{T}+A_{i} Q-B_{i} M_{j}-M_{j}^{T} B_{i}^{T} & \left(A_{i}-D_{k}\right) Q \\
Q\left(A_{i}-D_{k}\right)^{T} & -\rho^{2} I
\end{array}\right]<0,} \\
\text { for } i=1, \ldots, c, j=1, \ldots, c, i \neq j, k=1, \ldots, c r
\end{gathered}
$$

with $\rho>0$.

Proof. The state tracking error is given by

$$
e(t)=x_{R}(t)-x(t) \text {. }
$$


The following quadratic Lyapunov candidate function which is positive definite, has been used to verify the system stability and compute the feedback gains $K_{i}$ :

$$
V(e)=e^{T}(t) P e(t)+\frac{1}{\rho^{2}} \int_{0}^{t} x_{R}^{T}(\tau) x_{R}(\tau) d \tau .
$$

The system is asymptotically stable if we prove that $\dot{V}(e)<0$ :

$$
\begin{aligned}
& \dot{V}(e)=\dot{e}^{T} P e+e^{T} P \dot{e}+\frac{1}{\rho^{2}} x_{R}^{T} x_{R}, \\
& \dot{e}(t)=\sum_{i=1}^{c} \sum_{k=1}^{c r} h_{i}(z) \eta_{k}(z) \\
& \times\left[A_{i} e(t)+B_{i} u(t)+\left(A_{i}-D_{k}\right) x_{R}(t)\right], \\
& \dot{e}(t)=\sum_{i=1}^{c} \sum_{j=1}^{c} \sum_{k=1}^{c r} h_{i}(z) h_{j}(z) \eta_{k}(z) \\
& \times\left[A_{i} e(t)-B_{i}\left(K_{j} e\right)+\left(A_{i}-D_{k}\right) x_{R}(t)\right], \\
& \dot{V}(e)=\sum_{i=1}^{c} \sum_{j=1}^{c} \sum_{k=1}^{c r} h_{i}(z) h_{j}(z) \eta_{k}(z) \\
& \times\left[\left(A_{i}-B_{i} K_{j}\right) e(t)\right. \\
& \left.+\left(A_{i}-D_{k}\right) x_{R}(t)\right]^{T} P e \\
& +e^{T} P \sum_{i=1}^{c} \sum_{j=1}^{c} \sum_{k=1}^{c r} h_{i}(z) h_{j}(z) \eta_{k}(z) \\
& \times\left[\left(A_{i}-B_{i} K_{j}\right) e(t)\right. \\
& \left.+\left(A_{i}-D_{k}\right) x_{R}(t)\right] \\
& +\frac{1}{\rho^{2}} x_{R}^{T} x_{R}, \\
& \dot{V}(e)=\sum_{i=1}^{c} \sum_{j=1}^{c} \sum_{k=1}^{c r} h_{i}(z) h_{j}(z) \eta_{k}(z) \\
& \times\left(e^{T}\left(A_{i}-B_{i} K_{j}\right)^{T} P e+e^{T} P\left(A_{i}-B_{i} K_{j}\right) e\right. \\
& \left.+x_{R}^{T}\left(A_{i}-D_{k}\right)^{T} P e+e^{T} P\left(A_{i}-D_{k}\right) x_{R}\right) \\
& +\frac{1}{\rho^{2}} x_{R}^{T} x_{R}, \\
& \dot{V}(e)=\sum_{i=1}^{c} \sum_{k=1}^{c r} h_{i}^{2}(z) \eta_{k}(z) \\
& \times\left(e^{T}\left(A_{i}-B_{i} K_{i}\right)^{T} P e+e^{T} P\left(A_{i}-B_{i} K_{i}\right) e\right. \\
& +x_{R}^{T}\left(A_{i}-D_{i}\right)^{T} P e+e^{T} P\left(A_{i}-D_{k}\right) x_{R} \\
& \left.+\frac{1}{\rho^{2}} x_{R}^{T} x_{R}\right)
\end{aligned}
$$

$$
\begin{aligned}
+\sum_{\substack{i=1 \\
i \neq j}}^{c} \sum_{j=1}^{c} \sum_{k=1}^{c r} h_{i}(z) h_{j}(z) \eta_{k}(z) & \\
& \times\left(e^{T}\left(A_{i}-B_{i} K_{j}\right)^{T} P e\right. \\
& +e^{T} P\left(A_{i}-B_{i} K_{j}\right) e+x_{R}^{T}\left(A_{i}-D_{k}\right)^{T} P e \\
& \left.+e^{T} P\left(A_{i}-D_{k}\right) x_{R}+\frac{1}{\rho^{2}} x_{R}^{T} x_{R}\right) .
\end{aligned}
$$

We note in this analysis that the feedback gains and stability conditions will be transformed to an LMI problem. However, the inequality $\dot{V}(e)<0$ is guaranteed when the following inequalities are satisfied:

$$
\begin{aligned}
e^{T}\left(A_{i}-B_{i} K_{i}\right)^{T} P e & +e^{T} P\left(A_{i}-B_{i} K_{i}\right) e+x_{R}^{T}\left(A_{i}-D_{k}\right)^{T} P e \\
& +e^{T} P\left(A_{i}-D_{k}\right) x_{R}+\frac{1}{\rho^{2}} x_{R}^{T} x_{R}<0, \\
e^{T}\left(A_{i}-B_{i} K_{j}\right)^{T} P e & +e^{T} P\left(A_{i}-B_{i} K_{j}\right) e+x_{R}^{T}\left(A_{i}-D_{k}\right)^{T} P e \\
& +e^{T} P\left(A_{i}-D_{k}\right) x_{R}+\frac{1}{\rho^{2}} x_{R}^{T} x_{R}<0 .
\end{aligned}
$$

Using Schur complement [10], the inequalities given in (24) can be written as

$$
\begin{aligned}
& {\left[\begin{array}{cc}
A_{i}^{T} P+P A_{i}-P B_{i} K_{i}-K_{i}^{T} B_{i}^{T} P & P\left(A_{i}-D_{k}\right) \\
\left(A_{i}-D_{k}\right)^{T} P & -\rho^{2} I
\end{array}\right]<0,} \\
& {\left[\begin{array}{cc}
A_{i}^{T} P+P A_{i}-P B_{i} K_{j}-K_{j}^{T} B_{i}^{T} P & P\left(A_{i}-D_{k}\right) \\
\left(A_{i}-D_{k}\right)^{T} P & -\rho^{2} I
\end{array}\right]<0,} \\
& \text { for } i=1, \ldots, c, j=1, \ldots, c, i \neq j, k=1, \ldots, c r .
\end{aligned}
$$

Since coupled elements, such as $P B_{i} K_{i}$, have been enclosed in these inequalities, then we have BiLMIs inequalities. However, we must transform them to the LMIs using a congruence transformation by $\operatorname{diag}\left[\begin{array}{lll}P^{-1} & I & I\end{array}\right]$ to (29) and considering $Q=P^{-1}, M_{i}=K_{i} P^{-1}$, we obtain the following matrices in the LMI form:

$$
\begin{gathered}
{\left[\begin{array}{cc}
Q A_{i}^{T}+A_{i} Q-B_{i} M_{i}-M_{i}^{T} B_{i}^{T} & \left(A_{i}-D_{k}\right) Q \\
Q\left(A_{i}-D_{k}\right)^{T} & -\rho^{2} I
\end{array}\right]<0,} \\
{\left[\begin{array}{cc}
Q A_{i}^{T}+A_{i} Q-B_{i} M_{j}-M_{j}^{T} B_{i}^{T} & \left(A_{i}-D_{k}\right) Q \\
Q\left(A_{i}-D_{k}\right)^{T} & -\rho^{2} I
\end{array}\right]<0,}
\end{gathered}
$$


TABLE 1: Characteristics of the PV array panel.

$N_{p}=1$
$N_{s}=36$
$q=1.6 e-19 \mathrm{C}$
$A=1.92$
$E_{g}=1.1$
$T_{r}=298.18^{\circ} \mathrm{K}$
$T_{r}=25^{\circ} \mathrm{C}$
$I_{\text {or }}=9.579 e-6 \mathrm{~A}$
$V_{\text {co }}=27.4 \mathrm{~V}$
$R_{s}=0.09 \Omega$
$R_{\text {sh }}=100 \Omega$
$R_{\text {load }}=R_{L}=30 \Omega$
$P_{\text {max }}=61 \mathrm{~W}$
$I_{\text {sc }}=4.8 \mathrm{~A}$
$K_{I}=0.00171 \mathrm{~A} /{ }^{\circ} \mathrm{C}$
$F=10 \mathrm{KHz}$
$C_{2}=68 \mu \mathrm{F}$
$K=1.38 .10^{-23} \mathrm{~J} / \mathrm{K}$ (Boltzmann's constant)

\section{Simulation Results}

This section is reserved for presenting the main results. We use MATLAB to simulate the behavior of the energy conversion system. The main characteristics of the PV array panel are given by Table 1 .

The resolution of the LMIs gives the following matrices and feedback gains, respectively, $P, Q, K_{1}, K_{2}, K_{3}, K_{4}, K_{5}, K_{6}$, $K_{7}$, and $K_{8}$.

$$
\begin{aligned}
& P=100\left[\begin{array}{cccc}
78.9262 & -0.9022 & 0.5037 & -0.0002 \\
-0.9022 & 0.1748 & -0.0088 & -0.0000 \\
0.5037 & -0.0088 & 0.0056 & -0.0000 \\
-0.0002 & -0.0000 & -0.0000 & 0.0008
\end{array}\right] \\
& Q=\left[\begin{array}{cccc}
0.0003 & 0.0002 & -0.0266 & 0.0000 \\
0.0002 & 0.0623 & 0.0802 & 0.0004 \\
-0.0266 & 0.0802 & 4.3183 & -0.0007 \\
0.0000 & 0.0004 & -0.0007 & 12.1668
\end{array}\right] \text {, } \\
& K_{1}=-1.0 e+004\left[\begin{array}{llll}
-0.3951 & -0.0579 & -0.0008 & -4.5760
\end{array}\right] \text {, } \\
& K_{2}=-1.0 e+004\left[\begin{array}{llll}
-0.3661 & -0.0492 & -0.0035 & -4.5715
\end{array}\right] \text {, } \\
& K_{3}=-1.0 e+004\left[\begin{array}{llll}
-0.4047 & -0.0608 & -0.0009 & -4.5780
\end{array}\right], \\
& K_{4}=-1.0 e+004\left[\begin{array}{llll}
-0.3983 & -0.0543 & -0.0010 & -4.5786
\end{array}\right] \text {, } \\
& K_{5}=-1.0 e+004\left[\begin{array}{llll}
-0.3757 & -0.0522 & -0.0036 & -4.5735
\end{array}\right] \text {, } \\
& K_{6}=-1.0 e+004\left[\begin{array}{llll}
-0.3693 & -0.0457 & -0.0037 & -4.5741
\end{array}\right] \text {, } \\
& K_{7}=-1.0 e+004\left[\begin{array}{llll}
-0.4015 & -0.0644 & -0.0007 & -4.5754
\end{array}\right] \text {, } \\
& K_{8}=-1.0 e+004\left[\begin{array}{llll}
-0.3725 & -0.0557 & -0.0035 & -4.5710
\end{array}\right] \text {. }
\end{aligned}
$$

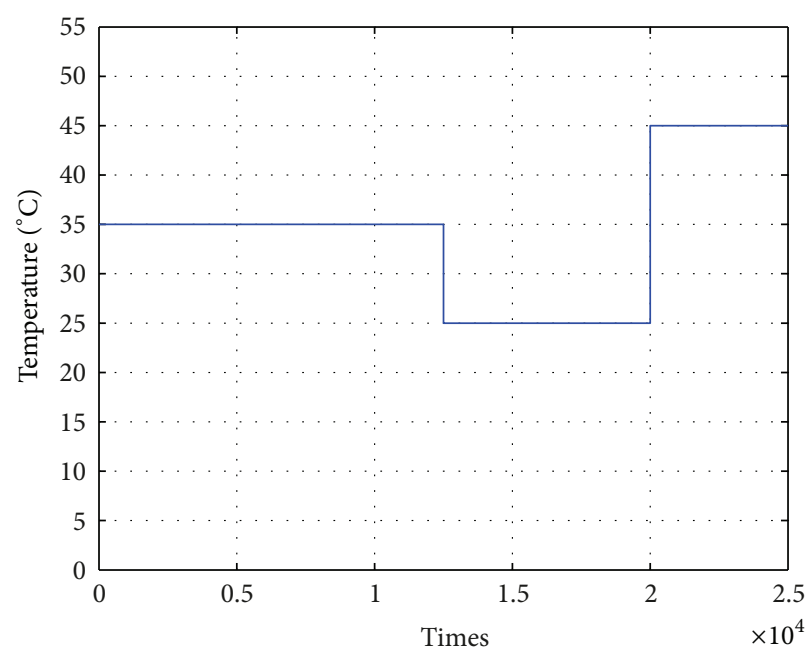

FIgURE 9: Evolution of temperature.

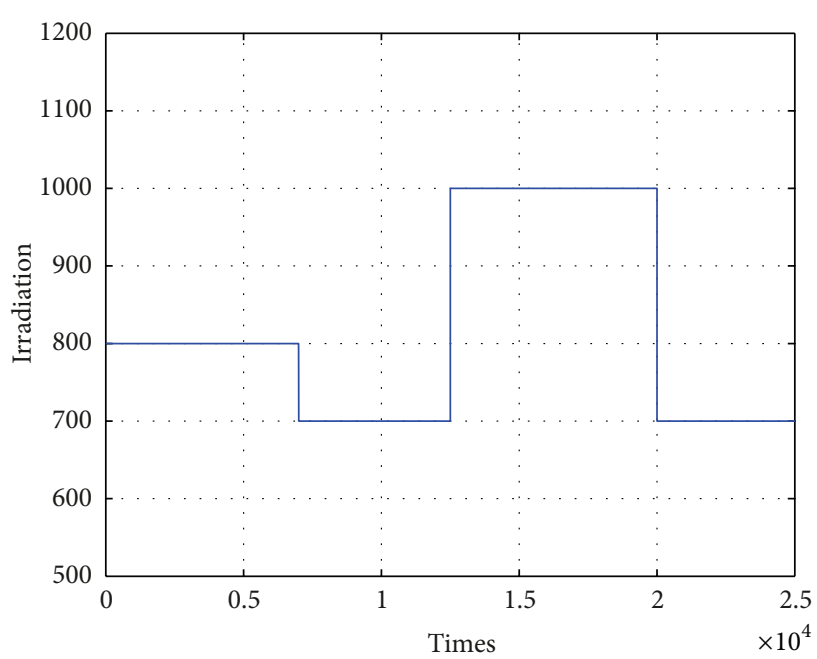

FIGURE 10: Evolution of irradiation.

To demonstrate the performance of the proposed MPPT control approach, we apply a sudden variation of temperature or solar irradiation as shown in Figures 9 and 10.

In Figure 9, we have applied a sudden change of temperature, although it is impossible to have a really dramatic change.

In this test, we have chosen four pairs of irradiation and temperature. We know that for each pair there exists only one optimal operating point which can be determined from the power-voltage characteristics of the PV array panel which is not always available for each pair $(G, T)$. It is important to mention that it is not possible to know the appropriate coordinates of the ideal optimal operating point $\left(V_{\mathrm{MPP}}, I_{\mathrm{MPP}}\right)$ for all pairs $(G, T)$ as there are infinitely of pairs $(G, T)$.

In Table 2, we give the ideal corresponding values $\left(V_{\mathrm{MPPR}}, I_{\mathrm{MPPR}}\right)$ of operating point for each pair of temperature 
TABLE 2: Coordinates for each operating point.

\begin{tabular}{lccccc}
\hline Temperature in ${ }^{\circ} \mathrm{C}$ & Irradiation $\left(\mathrm{w} \mathrm{m}^{-2}\right)$ & $V_{\text {MPPR }}(\mathrm{V})$ & $I_{\text {MPPR }}(\mathrm{A})$ & $V_{\text {MPP }}(\mathrm{V})$ & $I_{\text {MPP }}(\mathrm{A})$ \\
\hline 35 & 800 & 14.445 & 3.343 & 14.2494 & 3.2875 \\
35 & 700 & 14.519 & 2.940 & 14.2226 & 2.8805 \\
25 & 1000 & 14.92 & 4.16 & 14.9846 & 4.1457 \\
45 & 700 & 13.794 & 2.917 & 13.4918 & 2.8504 \\
\hline
\end{tabular}

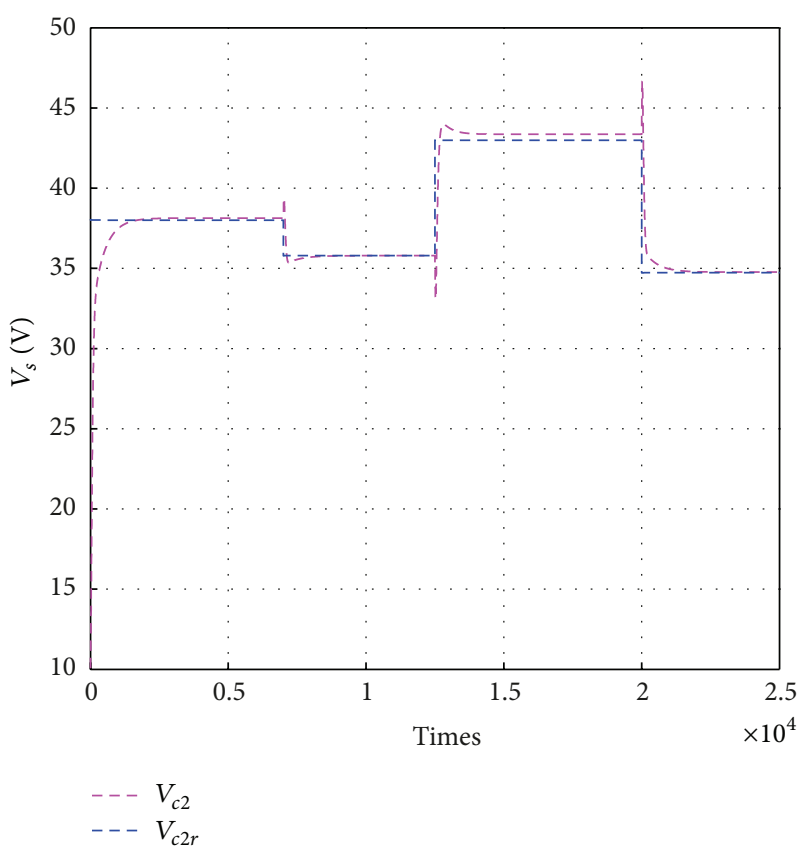

FIGURE 11: Evolution of output voltage.

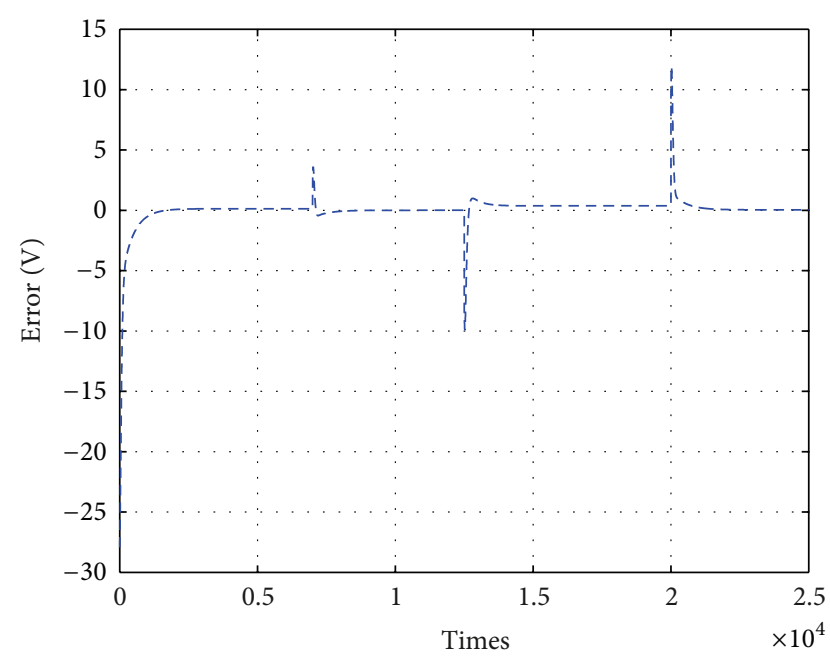

FIGURE 12: Evolution of error output voltage.

and irradiation and the computed values $\left(V_{\mathrm{MPP}}, I_{\mathrm{MPP}}\right)$ by our algorithm.

Figures 11, 12, 13, 14, 15, 16, 17, 18, and 19 show, respectively, the evolution of $V_{\text {MPP }}$ voltage,error of $V_{\text {MPP }}$ voltage, output

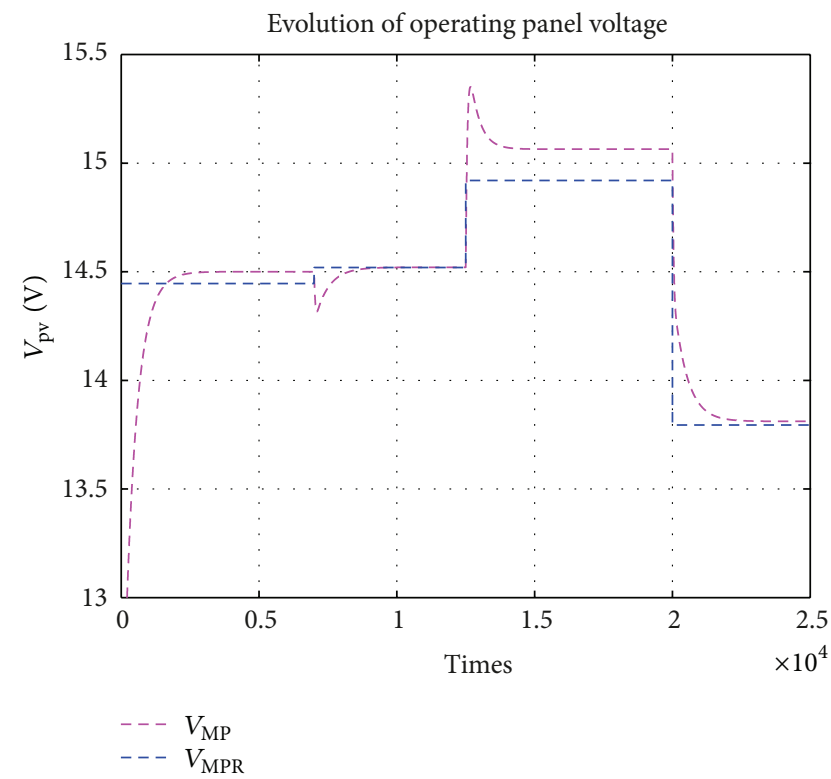

Figure 13: Evolution of the $V_{\text {MPP }}$ voltage.

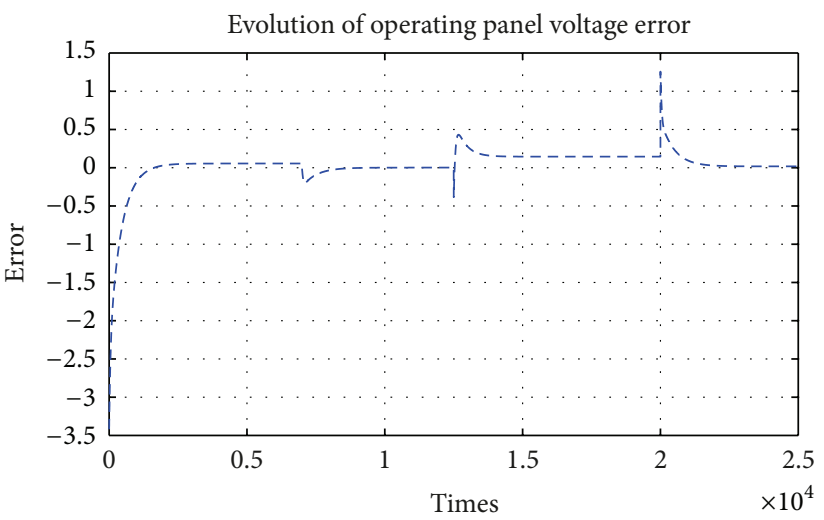

FIGURE 14: Evolution of error $V_{\text {MPP }}$ voltage.

voltage of converter, error of output voltage of converter, panel current, error of panel current, delivered power, error of delivered power, and the duty cycle.

In Figures $12,13,14,15,16,17$, and 18 , we observe momentary peaks; they are due to sudden and significant change in temperature and irradiation. The changes in temperature and irradiation are not made as that way given in Figures 9 and 10, but we have used it to show the performance of the proposed 


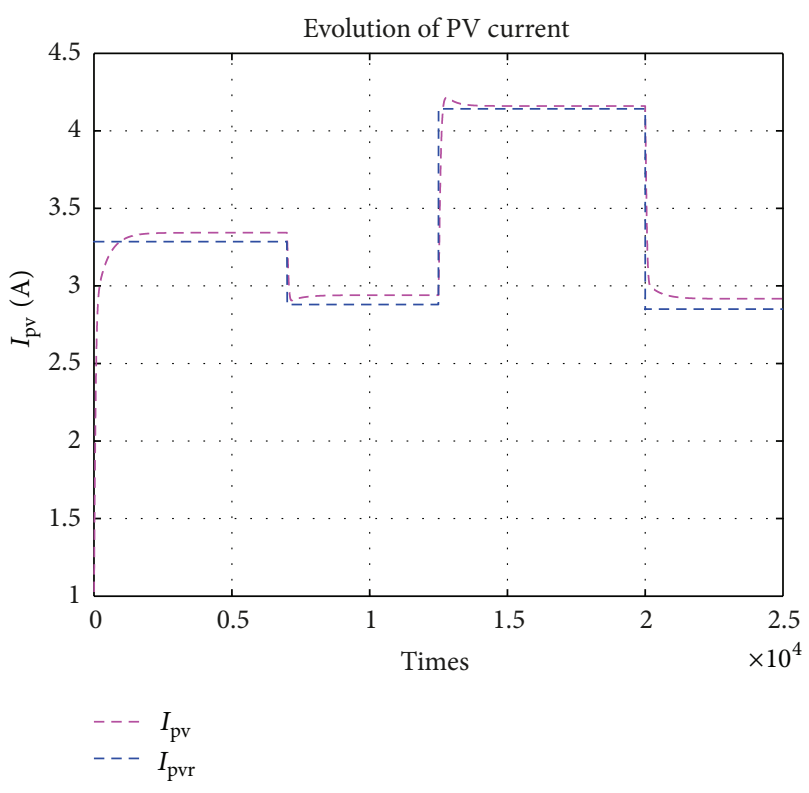

FIGURE 15: Evolution of the inductance current.

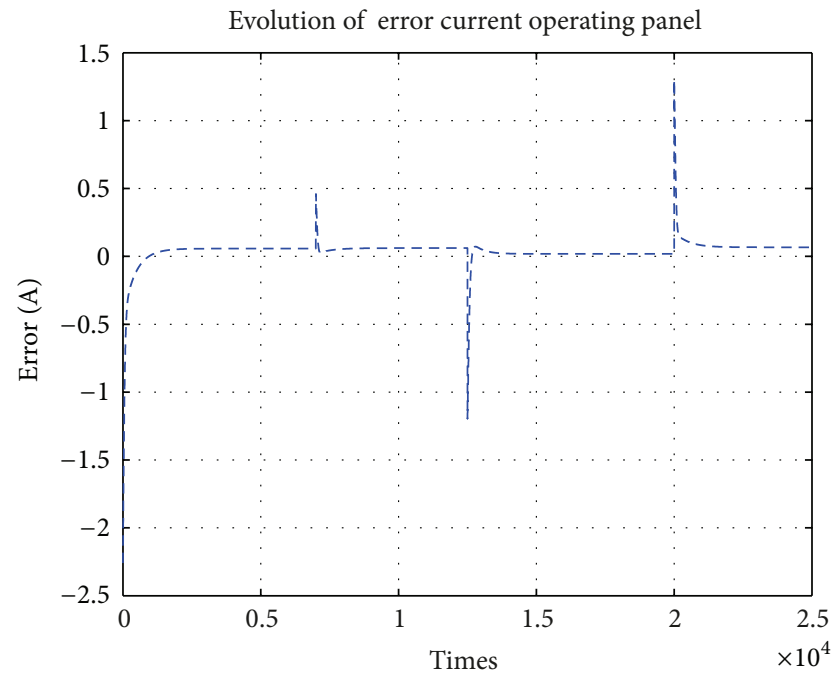

FIGURE 16: Evolution of the error inductance current.

algorithm. It is clear that at the steady state, the errors tend toward zero and the state variables reach the reference one. Also, it is visible that the computed coordinates, of optimal operating point, based on the proposed algorithm, are almost the same as the ideal optimal operating point. This analysis allows demonstrating the performance of the proposed algorithm.

\section{Conclusion}

In this paper, a new algorithm strategy based on the augmented Takagi-Sugeno type fuzzy system has been proposed

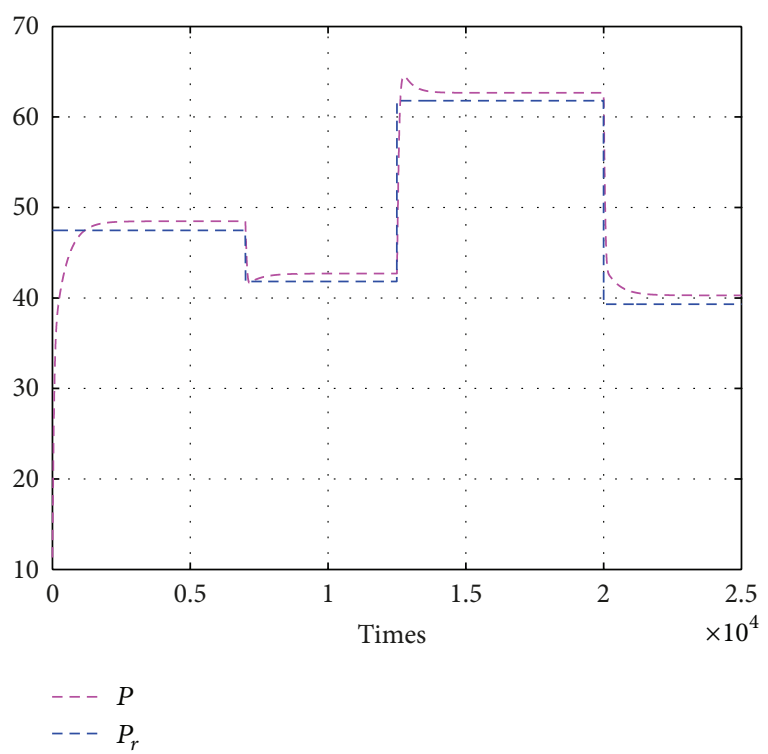

Figure 17: Evolution of the power.

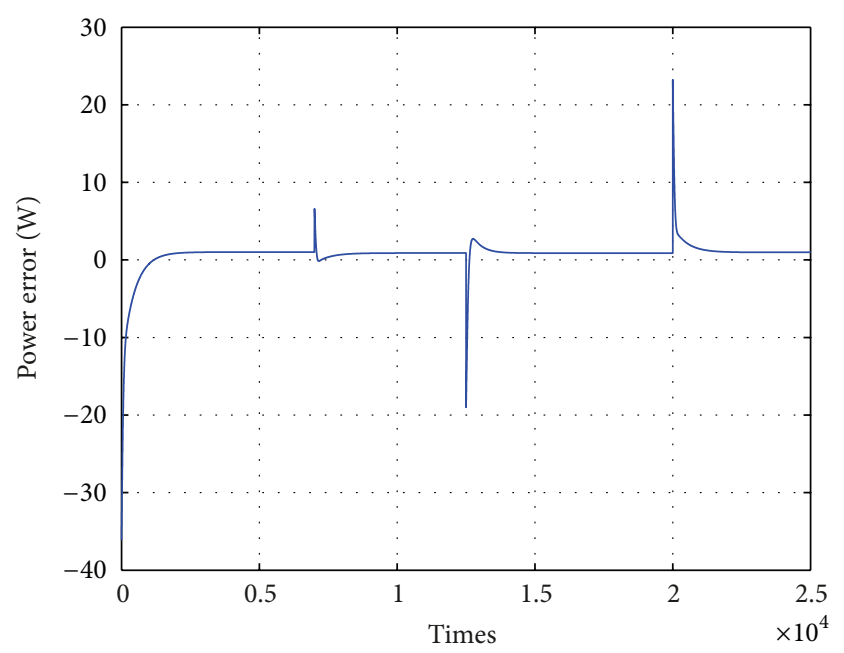

FIgURE 18: Evolution of the error power.

for the MPPT of a PV energy system. All the PV system has been modeled by T-S fuzzy system. Based on the measurement of temperature and irradiation, we deduce the coordinates of the desired optimal operating point which corresponds to the maximum power.

The MPPT algorithm is based on an augmented T-S fuzzy model and PDC method. The controller parameters have been computed based on the LMI tools. The stability of system has been proved based on Lyapunov approach. The simulation results show that the proposed algorithm tracks quickly the optimal operating point despite sudden variations of temperature and irradiation. 


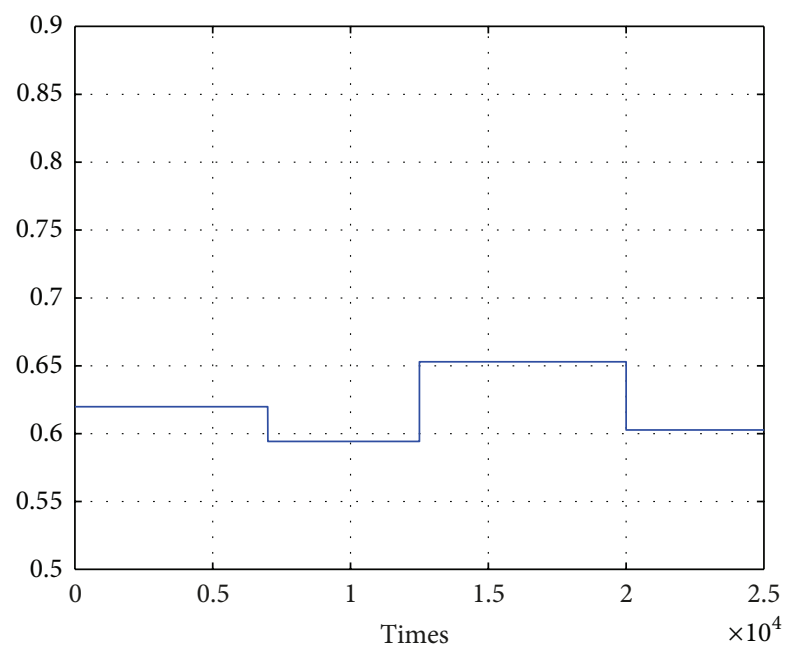

Figure 19: Evolution of the duty cycle.

\section{Conflict of Interests}

The authors declare that there is no conflict of interests regarding the publication of this paper.

\section{References}

[1] A. Chermitti, O. Boukli-Hacene, and B. Mohamed, "Improvement of the "perturb and observe" MPPT algorithm in a photovoltaic system under rapidly changing climatic conditions," International Journal of Computer Applications, vol. 56, no. 12, pp. 5-10, 2012.

[2] J. J. Nedumgatt, K. B. Jayakrishnan, S. Umashankar, D. Vijayakumar, and D. P. Kothari, "Perturb and observe MPPT algorithm for solar PV systems-modeling and simulation," in Proceedings of the Annual IEEE India Conference: Engineering Sustainable Solutions (INDICON '11), Hyderabad, India, December 2011.

[3] A. P. K. Yadav, S. Thirumaliah, and G. Haritha, "Comparison of MPPTalgorithms for DC-DC converters based PV systems," International Journal of Advanced Research in Electrical, Electronics and Instrumentation Engineering, vol. 1, no. 1, pp. 18-23, 2012.

[4] M. Azzouzi, "Comparaison between MPPT P\&O and MPPT fuzzy controls in optimizing the photovoltaic generator," International Journal of Advanced Computer Science and Applications, vol. 3, no. 12, pp. 57-62, 2012.

[5] S. Gomathy, S. Saravanan, and S. Dr. Thangave, "Design and implementation of maximum power point tracking (MPPT) algorithm for a standalone PV system," International Journal of Scientific \& Engineering Research, vol. 3, no. 3, pp. 2229-5518, 2012.

[6] G. Balasubramanian and S. Singaravelu, "Fuzzy logic controller for the maximum power point tracking in photovoltaic system," International Journal of Computer Applications, vol. 41, no. 12, pp. 22-28, 2012.

[7] L. Jie and C. Ziran, "Research on the MPPT algorithms of photovoltaic system based on PV neural network," in Proceedings of the Chinese Control and Decision Conference (CCDC '11), pp. 1851-1854, Mianyang, China, May 2011.
[8] S. Singer, B. Rozenshtein, and S. Surazi, "Characterization of PV array output using a small number of measured parameters," Solar Energy, vol. 32, no. 5, pp. 603-607, 1984.

[9] H. O. Wang, K. Tanaka, and M. Griffin, "Parallel distributed compensation of nonlinear systems by Takagi-Sugeno fuzzy model," in Proceedings of the IEEE International Conference on Fuzzy Systems, pp. 531-538, March 1995.

[10] L. El Ghaoui and G. Scorletti, "Control of rational systems using linear-fractional representations and linear matrix inequalities," Automatica, vol. 32, no. 9, pp. 1273-1284, 1996. 


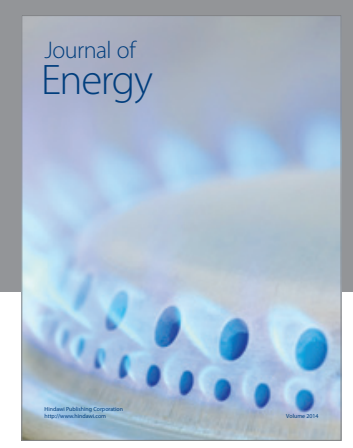

Journal of

Industrial Engineering
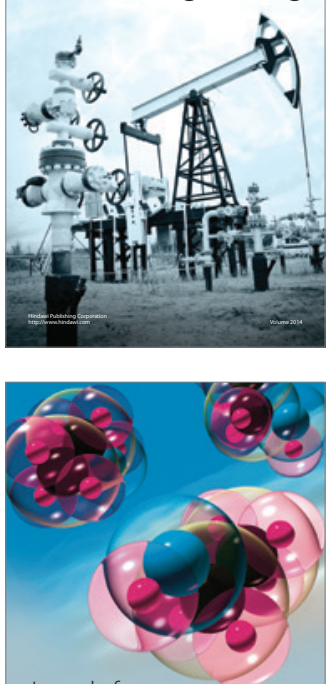

Fuels
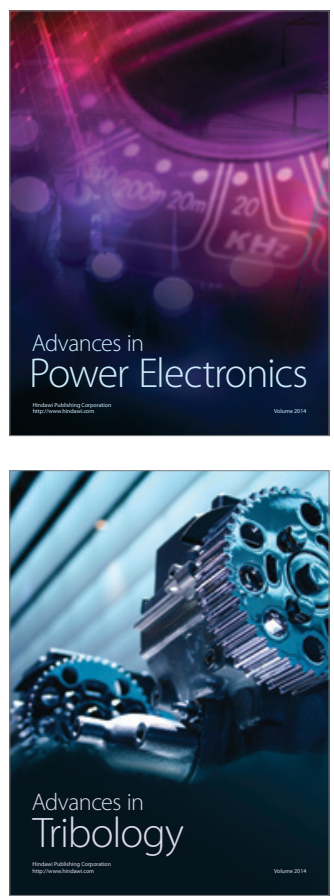

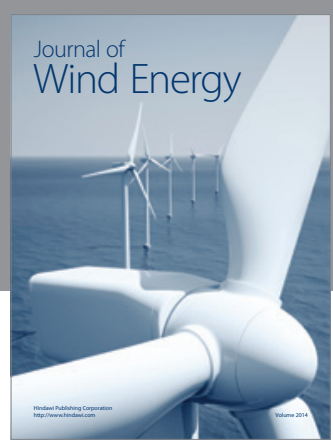

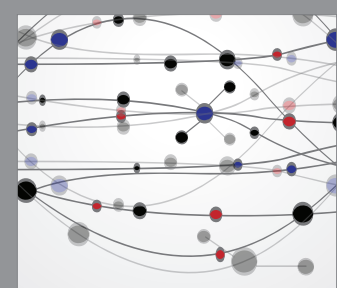

The Scientific World Journal

Submit your manuscripts at http://www.hindawi.com

Journal of

Structures
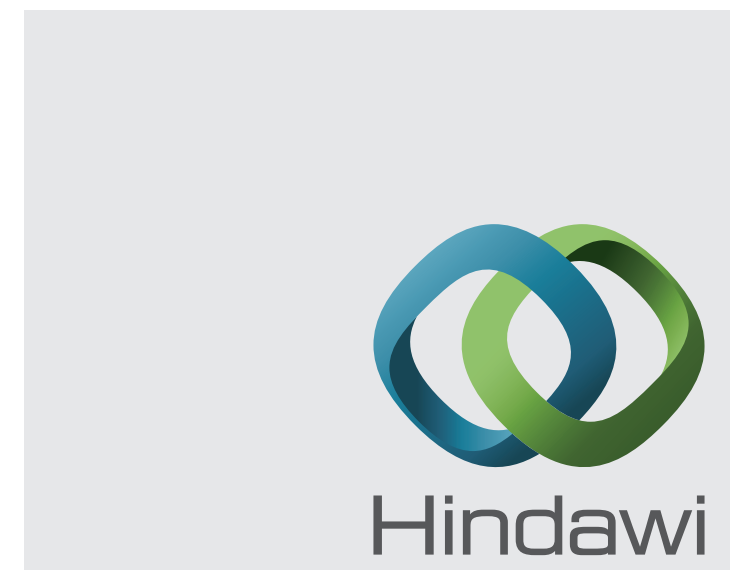

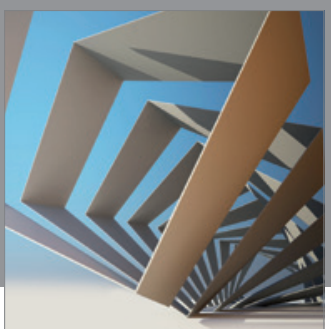

Rotating

Machinery
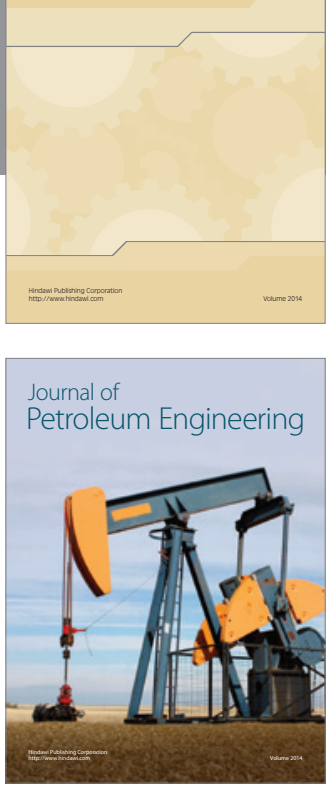

Journal of

Solar Energy
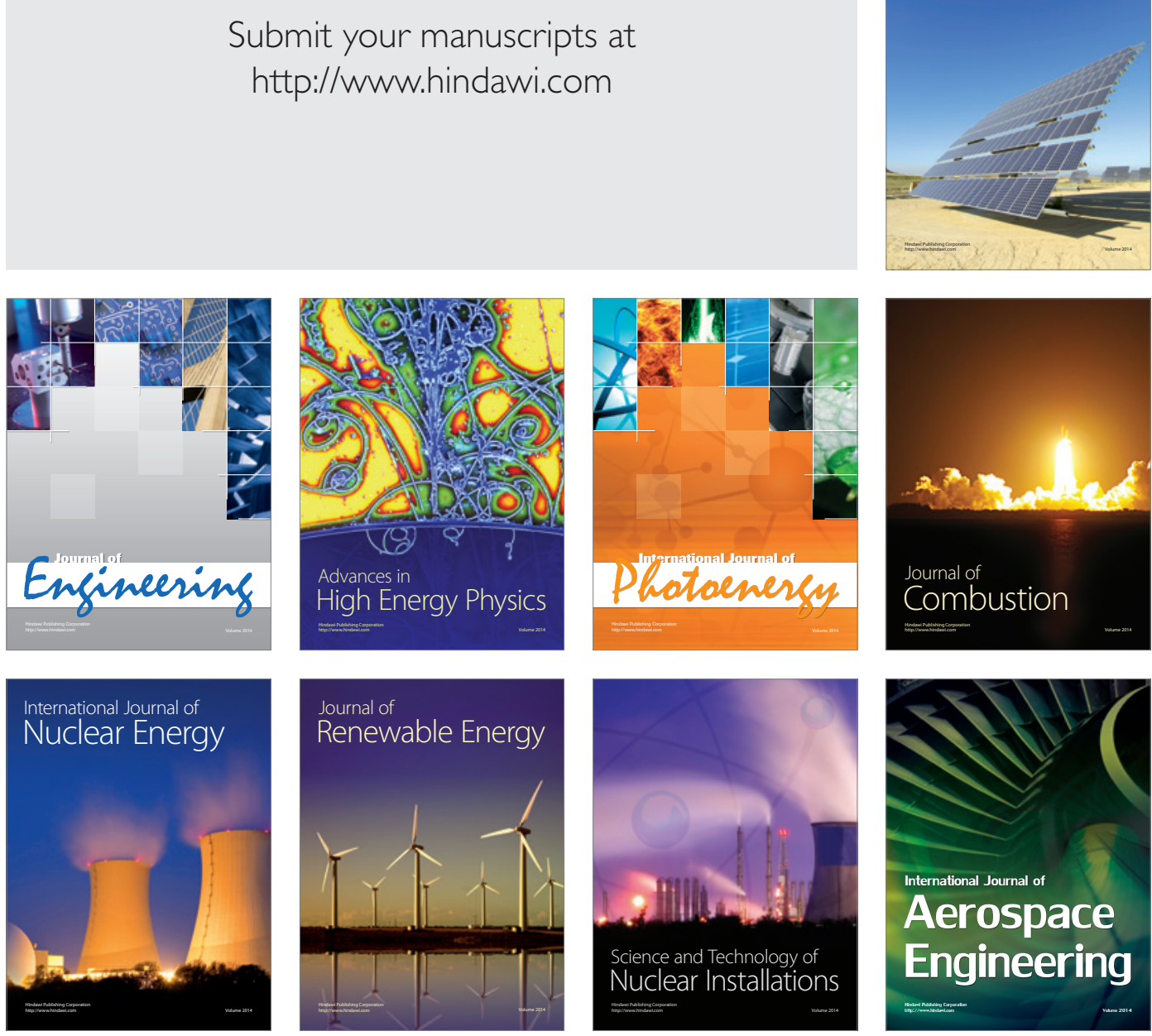